\title{
SPECTRAL METHOD FOR MULTIPLEXED PHASE RETRIEVAL AND APPLICATION IN OPTICAL IMAGING IN COMPLEX MEDIA
}

\author{
Jonathan Dong Florent Krzakala $^{2} \quad$ Sylvain Gigan ${ }^{1}$ \\ ${ }^{1}$ Laboratoire Kastler Brossel, ENS-Université PSL, CNRS, Sorbonne Université, \\ Collège de France, 24 rue Lhomond, 75005 Paris, France \\ ${ }^{2}$ Laboratoire de Physique Statistique, ENS-Université PSL, CNRS, \\ Sorbonne Université, 75005, Paris, France
}

\begin{abstract}
We introduce a generalized version of phase retrieval called multiplexed phase retrieval. We want to recover the phase of amplitude-only measurements from linear combinations of them. This corresponds to the case in which multiple incoherent sources are sampled jointly, and one would like to recover their individual contributions. We show that a recent spectral method developed for phase retrieval can be generalized to this setting, and that its performance follows a phase transition behavior. We apply this new technique to light focusing at depth in a complex medium. Experimentally, although we only have access to the sum of the intensities on multiple targets, we are able to separately focus on each ones, thus opening potential applications in deep fluorescence imaging and light delivery.
\end{abstract}

Index Terms - Phase retrieval, multiplexed phase retrieval, matrix factorization, random matrix theory, imaging in complex media

\section{INTRODUCTION}

To recover a complex-valued object from amplitude measurements only is a computational problem known as phase retrieval. The first iterative algorithms date back to the works of Gerchberg and Saxton [1] followed by Fienup [2], with very successful applications in optics such as the measurement and correction of aberrations in the space telescope Hubble in 1990 [3]. Since then, phase retrieval algorithms have been applied in a number of domains including microscopy [4], astronomy [5], acoustics [6], and quantum mechanics [7].

Recently, new concepts have been introduced to solve the phase retrieval problem. Semi-definite programming algorithms [8, 9] are guaranteed to converge but computationally and memory intensive, while non-linear optimization methods [10-12] are very efficient for real-world applications. In all these algorithms, there is an initialization step, which consists in finding a good initial guess for subsequent iterative algorithms. For this purpose, a spectral method has been proposed [10, 11], setting the initial estimate to be the principal eigenvector of a certain matrix (the eigenvector corresponding to the largest eigenvalue). One can even prove that this estimate becomes very close to the optimal solution when the number of measurements is large enough, provided the measurements are random and independent.

We introduce here the more difficult problem of multiplexed phase retrieval. Instead of measuring the intensity of a signal, we measure the linear combination of the intensities of several signals. This problem arises for instances when measurements acquired by a physical detector come from several indistinguishable sources. By solving the multiplexed phase retrieval problem, one can unmix the signal from each source and ideally retrieve information about each of the sources.

In this paper, we show that this multiplexed phase retrieval problem is well suited for spectral methods. When the number of measurement is large enough, the leading eigenvector retrieves the signal from the brightest source, the second leading eigenvector corresponds to the second brightest source, and so on. We present numerical results that point to a phase transition behavior, similar to the one seen in many estimation problems with spiked matrices [13-18] in random matrix theory.

We then experimentally apply the method to complex media optics [19]: we show in a proof-of-concept experiment that, by solving a multiplexed phase retrieval problem, we are able to disentangle the signals coming from multiple sources, that could for instance be multiple fluorescent beads buried inside a diffusive material.

The paper is organized as follows: In Section 2, we introduce the multiplexed phase retrieval problem. To solve it, we present a spectral algorithm in Section 3 and numerical results in Section 4. Finally, we present an application for imaging in complex media in Section 5 .

\section{MULTIPLEXED PHASE RETRIEVAL}

We will first introduce the phase retrieval problem as formulated by [11], followed by the multiplexed version. We con- 

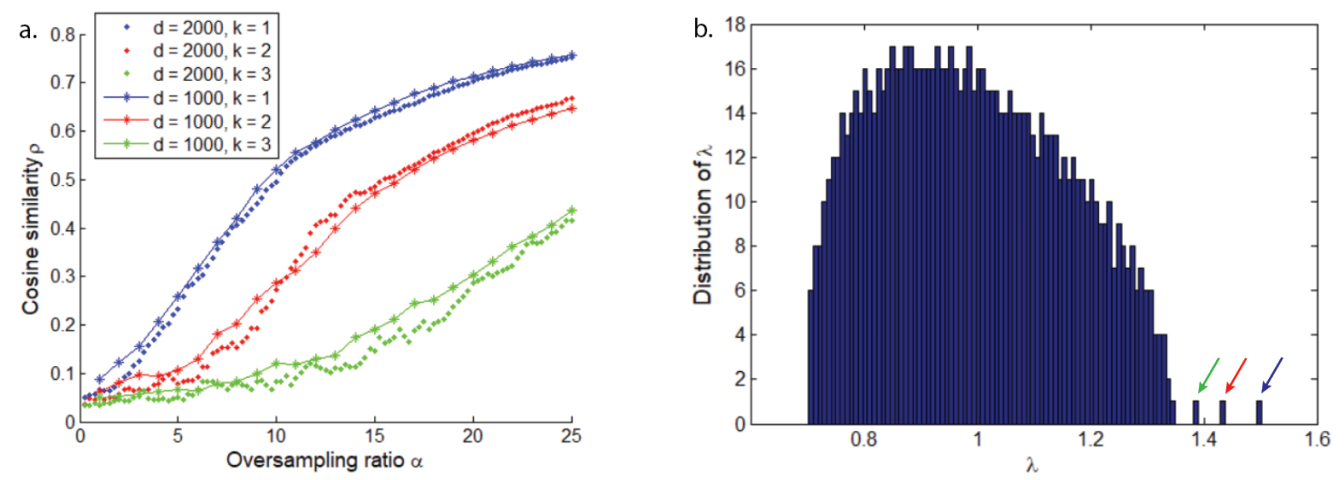

Fig. 1. Phase transitions in spectral methods for multiplexed phase retrieval. a. Cosine similarity $\rho$ as a function of the oversampling ratio $\alpha$ (higher is better). Three sources are present with respective weights $\frac{5}{12}$ (in blue), $\frac{4}{12}$ (in red), $\frac{3}{12}$ (in green). Dots correspond to $d=2000$ and lines to $d=1000$, each point is a mean of 10 realizations. b. Histogram of eigenvalues of the Weighted Covariance Matrix $\boldsymbol{Y}$ for $\alpha=50$.

sider the following set of quadratic equations:

$$
y_{i}=\left|\boldsymbol{a}_{i}^{*} \boldsymbol{x}\right|^{2}, \quad i \in\{1, \ldots, n\}
$$

where $\boldsymbol{x} \in \mathbb{C}^{d}$ is an unknown vector, $\boldsymbol{a}_{i} \in \mathbb{C}^{d}$ are known sampling vectors, and $\boldsymbol{y}_{i} \in \mathbb{R}$ are measured intensities. Without the modulus square operation, this set of equations would boil down to a linear regression. Here, we need to retrieve the phase of $\boldsymbol{a}_{i}^{*} \boldsymbol{x}$ in order to find $\boldsymbol{x}$. Let us define the sampling matrix $\boldsymbol{A}=\left[\boldsymbol{a}_{1}^{*}, \ldots, \boldsymbol{a}_{n}^{*}\right] \in \mathbb{C}^{n \times d}$.

We will consider the case where $\boldsymbol{A}$ is a random matrix: every component is an independent random variable, following for example a complex gaussian law: $a_{i, j} \sim_{i . i . d}$. $\mathcal{C N}\left(0, \frac{1}{d}\right)$. Thus, every sampling vector $\boldsymbol{a}_{i}$ is an independent realization of a random vector $\boldsymbol{a}$. Reconstruction performance for this random model has been the subject of extensive theoretical studies [17, [18]. One important parameter is the oversampling ratio $\alpha=\frac{n}{d}$, which quantifies the difficulty of the problem. A higher oversampling ratio corresponds to a simpler phase retrieval problem, as we acquire more information about $\boldsymbol{x}$.

In multiplexed phase retrieval, we want to retrieve several normalized orthogonal vectors $\boldsymbol{x}_{1}, \ldots, \boldsymbol{x}_{K}$ from a linear combination of intensity measurements:

$$
y_{i}=\sum_{k=1}^{K} \lambda_{k}\left|\boldsymbol{a}_{i} \boldsymbol{x}_{k}\right|^{2}, \quad i \in\{1, \ldots, n\}
$$

For $k=1, \ldots, K$, each signal $\boldsymbol{x}_{k}$ corresponds to a source with strength $\lambda_{k}$. For normalization, we will assume that $\sum_{k} \lambda_{k}=1$. In optics, a sum of intensities is linked with incoherence, it occurs for instance when several incoherent sources are present. Fundamentally, multiplexed phase retrieval tackles the problem when these sources are physically indistinguishable as they are measured using the same sampling matrix. This situation arises in complex media optical imaging as explained in section 5
The multiplexed phase retrieval problem is not always solvable. We can write the measured intensities as:

$$
y_{i}=\boldsymbol{a}_{i}^{*} \boldsymbol{M} \boldsymbol{a}_{i}, \quad i \in\{1, \ldots, n\}
$$

This matrix $\boldsymbol{M}=\sum_{k=1}^{K} \lambda_{k} \boldsymbol{x}_{k} \boldsymbol{x}_{k}^{*}$ defines the measurements $\boldsymbol{y}$, so the best one can do is to retrieve $\boldsymbol{M}$, and from there recover the individual signals $\boldsymbol{x}$. This is only possible if $\boldsymbol{M}$ has no degenerate eigenvalue that would mix the eigenvectors, i.e. if all the $\lambda_{k}$ are pairwise distinct. Note also that orthogonality between the $\boldsymbol{x}_{k}$ is important as the eigenvectors computed by Support Vector Decomposition form an orthonormal basis.

\section{SPECTRAL METHOD}

To solve this multiplexed phase retrieval problem, we adapt the spectral method introduced in [17, 18]. We construct the same weighted covariance matrix and prove that the first few eigenvectors will retrieve each source separately.

We define the following weighted covariance matrix (WCM):

$$
\boldsymbol{Y}=\frac{1}{n} \sum_{i=1}^{n} y_{i} \boldsymbol{a}_{i} \boldsymbol{a}_{i}^{*}
$$

This WCM is a sum of rank 1 matrices constructed from the sampling vectors, ponderated by the intensity measured for each sampling vector. Without these weights, this matrix would correspond to an empirical estimate of the covariance matrix of the random vector $\boldsymbol{a}$, and contain no information about $\boldsymbol{x}_{k}$ for $k=1, \ldots, K$.

Informally, when $\boldsymbol{a}_{i}$ is aligned with a certain $\boldsymbol{x}_{k}, \boldsymbol{y}_{i}$ is higher, so that the WCM is biased towards every $\boldsymbol{x}_{k}$. This intuitive argument can be formalized by observing that in the limit $n$ going to infinity, $\boldsymbol{Y}$ converges towards the expected value of $y \boldsymbol{a} \boldsymbol{a}^{*}$, thanks to the Central Limit Theorem. This asymptotic limit can be computed explicitly by developing 
$\boldsymbol{Y}$ as a polynomial of order 4 in the components of $\boldsymbol{a}$ and computing the expected value of each term separately. When every component of the sampling matrix $\boldsymbol{A}$ follows a complex gaussian distribution, this expected value can be expressed as:

$$
\overline{\boldsymbol{Y}}=\boldsymbol{M}+\boldsymbol{I}
$$

Hence, for a high enough oversampling ratio, the leading eigenvectors of the WCM are going to align with the eigenvectors of $\boldsymbol{M}$ with eigenvalues $\lambda_{k}+1$, while $d-K$ remaining eigenvalues will converge to 1 and form a bulk distribution in Fig. 1 .

One may wonder how many measurements one needs to perform in order to recover $M$ using this spectral method. In [11], the authors have observed a phase transition when applying this spectral method in the non-multiplexed phase retrieval problem. When the oversampling ratio $\alpha$ is below a critical value, the leading eigenvectors of $\boldsymbol{y}$ are random and contain no information about $M$. Above this critical value, spectral methods retrieve a coarse estimate of $M$ that converges towards the solution as $\alpha$ grows. This empirical observation has been confirmed for phase retrieval in a theoretical study in [17]. In the next section, we will show numerical results supporting a phase transition in the multiplexed case as well.

\section{RESULTS}

In order to evaluate the performance of the previously introduced spectral method in the multiplexed case, we consider the cosine similarity function: $\rho(\boldsymbol{x}, \boldsymbol{y})=\frac{\left|\boldsymbol{x}^{*} \boldsymbol{y}\right|}{\|x\|\|y\|}$. It corresponds to the absolute value of the cosine between the two vectors $\boldsymbol{x}$ and $\boldsymbol{y}$. As such, this quantity is maximal and equal to 1 when $\boldsymbol{x}$ and $\boldsymbol{y}$ are completely aligned, and tends to zero when $\boldsymbol{x}$ and $\boldsymbol{y}$ are uncorrelated.

Figure $1 \mathrm{l}$ shows the performance of the spectral algorithm as a function of the oversampling parameter $\alpha$. Three vectors $\boldsymbol{x}_{1}, \boldsymbol{x}_{2}$, and $\boldsymbol{x}_{3}$ are sampled using a gaussian random matrix $\boldsymbol{A}$ and their intensities are combined with coefficients $\lambda_{1}=\frac{5}{12}, \lambda_{2}=\frac{4}{12}$, and $\lambda_{3}=\frac{3}{12}$. Curves corresponding to $d=1000$ and $d=2000$ are superposed, showing that the quantity of interest is indeed the oversampling parameter $\alpha$. We retrieve sources one by one from the brightest to the weakest. We believe that there is a succession of three phase transitions, that are smoothed here due to finite size effects.

The eigenvalue distribution of the WCM behaves like a spiked random matrix. When $\alpha$ is below the critical value for all the phase transitions, the eigenvalues of $\boldsymbol{Y}$ lie in a compact support and form a bulk distribution, which is typical for random matrices. During each phase transition, a single eigenvalue comes out and form a spike in the spectrum of $\boldsymbol{Y}$. When $\alpha$ is large, we see three spikes isolated from the remaining eigenvalues. Hence, looking at the spectrum, we are able to count the number of sources $K$ in the multiplexed phase retrieval problem.

\section{IMAGING IN COMPLEX MEDIA}

When light propagates in complex media like biological tissues, it encounters many refractive index inhomogeneities and gets scattered multiple times along propagation. This prevents imaging in depth: ballistic light is exponentially attenuated, which limits the penetration depth of conventional microscopy.

Using techniques of wavefront shaping [20, 21], it is now possible to modulate the incoming light precisely in order to form a focus deep inside the scattering medium. These techniques typically use iterative optimization using a feedback signal from a target in order to achieve a focus. It has been in particular shown that by optimizing the incident wavefront of a laser on a scattering medium containing a single buried fluorescent bead, one can focus on it by optimizing the total linear fluorescence signal [22]. However, when multiple fluorescent sources are present at depth in a scattering medium, their signals are summed and mixed on the detector, and they cannot be located nor separated. It is possible to harness nonlinearities to form a focus [23], but these methods are not applicable to linear fluorescence. We would like to distinguish them to focus on each of them individually. This task can be formulated as a multiplexed phase retrieval problem.

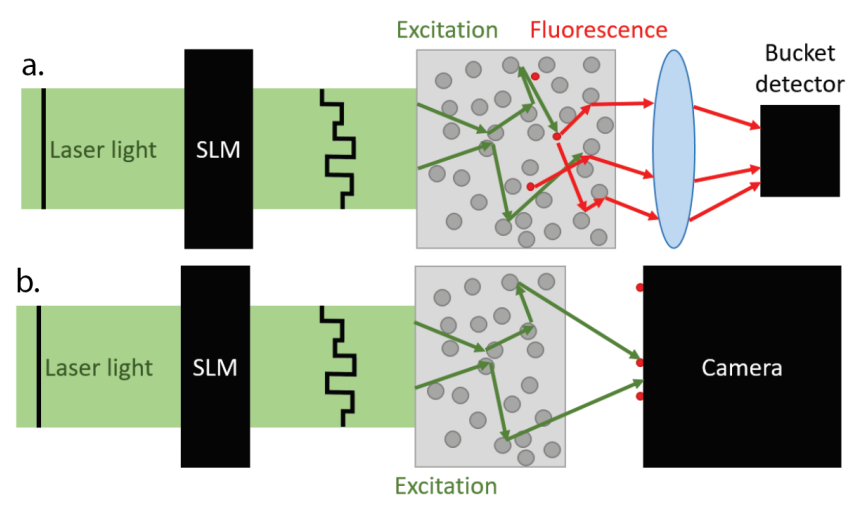

Fig. 2. Focusing light in a scattering media using linear fluorescence signals. a. When multiple fluorescent beads are placed in a complex media, their responses are summed on a bucket detector. b. To study how to focus on each target individually, we sum on a camera the excitation intensity after propagation in a complex media.

The experimental setup is presented in Figure 2b: excitation light from a laser (Coherent Sapphire 532-50 CW) is modulated by a Spatial Light Modulator (SLM, Holoeye Pluto-2 NIR), then propagates through a layer of white paint where it gets scattered multiple times. We place a camera (Basler acA1920 - 40um) after the scattering medium and observe a complex interference pattern called speckle.

To show the correspondence, we denote by $\boldsymbol{a}_{i}$ the phase pattern displayed on the SLM. We choose two given points 
a.

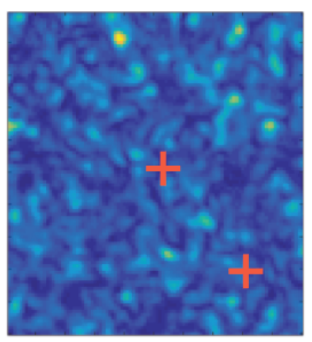

b.
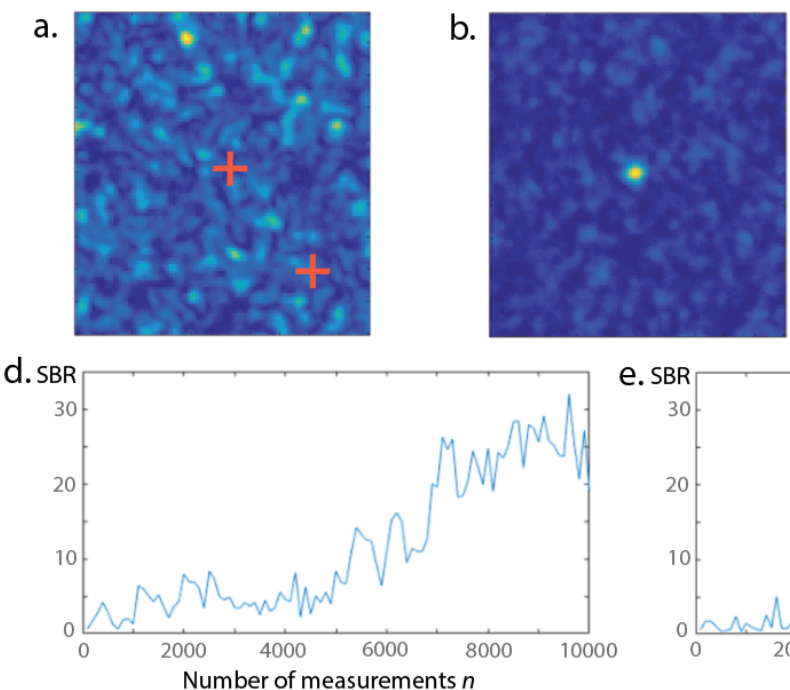

e. SBR

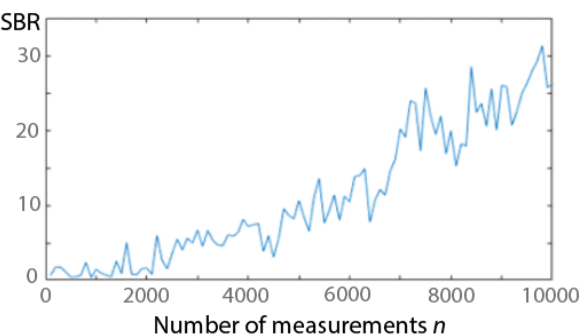

Fig. 3. Experimental application of the spectral method in complex media optics. a. Initial speckle pattern, resulting from light propagation in complex media in the absence of wavefront shaping. We simulate the linear fluorescence signals from targets at the two tagged positions with weights $\lambda_{1}=1$ and $\lambda_{2}=0.7$. b, c. Focus obtained by displaying the phase of the first (b) and second (c) eigenvector of the spectral method. d, e. Signal-to-Background Ratio for the first (d) and second (e) focus as the number of measurements $n$ increases.

on the camera after the scattering medium, and thanks to the Transmission Matrix formalism [24] that comes from the linearity of electric field propagation, the incident electric field on these two points can be written as $\boldsymbol{a}_{i}^{*} \boldsymbol{x}_{1}$ and $\boldsymbol{a}_{i}^{*} \boldsymbol{x}_{2}$ respectively, characterized by two vectors $\boldsymbol{x}_{1}$ and $\boldsymbol{x}_{2}$. Recovering them allows to focus light on each point individually. Fluorescent targets respond proportionally to the excitation intensity, which is the modulus square of the incident electric fields. In the end, we simulate the presence of two fluorescent beads of different brightness, by summing the intensities on these two positions with weights $\lambda_{1}=1$ and $\lambda_{2}=0.7$. We thus collect a signal obeying Equation (2).

To solve this multiplexed phase retrieval problem, we then send random phase patterns on the SLM and record the corresponding total fluorescence intensity. In this case, the sampling matrix $\boldsymbol{A}$ is a phase-only random matrix. The dimension of the SLM pattern is $d=256$ and the maximal number of measurements is $n=10^{\prime} 000$. Applying the previous spectral method, we obtain estimates of $\boldsymbol{x}_{1}$ and $\boldsymbol{x}_{2}$ from the WCM, that we can subsequently display on the SLM, and verify whether they correspond to focusing light at the positions where the beads are supposed to be, as shown in Figure 3. The oversampling ratio $\alpha=\frac{n}{d}$ is very large to compensate for experimental noise.

We see in Figure 3 experimental results, demonstrating that we are able to focus light on both targets individually with a Signal-to-Background Ratio (SBR) of 25. We believe that a higher SBR is reachable by improving the speed and stability of the experimental setup. In this first experiment, one exper- iment takes approximately one hour due to the speed of the SLM and the SBR is probably limited by the stability of the scattering medium. For the same reason, phase transitions appear smoothed because of these experimental considerations.

\section{CONCLUSION}

We introduced the multiplexed phase retrieval problem, which arises when multiple signals are incoherently summed. To solve this new task, we applied a spectral method recently designed for phase retrieval initialization. By constructing a Weighted Covariance Matrix and computing its leading eigenvectors, we are able to retrieve the different signals individually. The performance of this algorithm exhibits multiple phase transitions as we increase the number of measurements, recovering each signal one by one. We showed that this framework can be applied to imaging in complex media. Other potential applications where one would like to distinguish between different sources include opto-genetics, photoacoustics and multi-wavelength imaging.

\section{ACKNOWLEDGEMENTS}

We thank Leonie Muggenthaler and Romain Couillet for fruitful discussions. SG acknowledges funding from the ERC under the European Union 7th Framework Program Grant Agreements 724473 , \& FK from the ANR-PAIL. 


\section{References}

[1] RW Gerchberg and WO Saxton, "Untitled," Optik, vol. 35 , pp. 237, 1972.

[2] James R Fienup, "Phase retrieval algorithms: a comparison," Applied optics, vol. 21, no. 15, pp. 2758-2769, 1982.

[3] James R Fienup, Joseph C Marron, Timothy J Schulz, and John H Seldin, "Hubble space telescope characterized by using phase-retrieval algorithms," Applied optics, vol. 32, no. 10, pp. 1747-1767, 1993.

[4] Jianwei Miao, Tetsuya Ishikawa, Qun Shen, and Thomas Earnest, "Extending x-ray crystallography to allow the imaging of noncrystalline materials, cells, and single protein complexes," Annu. Rev. Phys. Chem., vol. 59, pp. 387-410, 2008.

[5] C Fienup and J Dainty, "Phase retrieval and image reconstruction for astronomy," Image Recovery: Theory and Application, vol. 231, pp. 275, 1987.

[6] Radu Balan, Pete Casazza, and Dan Edidin, "On signal reconstruction without phase," Applied and Computational Harmonic Analysis, vol. 20, no. 3, pp. 345-356, 2006.

[7] John V Corbett, "The pauli problem, state reconstruction and quantum-real numbers," Reports on Mathematical Physics, vol. 57, pp. 53-68, 2006.

[8] Emmanuel J Candes, Thomas Strohmer, and Vladislav Voroninski, "Phaselift: Exact and stable signal recovery from magnitude measurements via convex programming," Communications on Pure and Applied Mathematics, vol. 66, no. 8, pp. 1241-1274, 2013.

[9] Irène Waldspurger, Alexandre d'Aspremont, and Stéphane Mallat, "Phase recovery, maxcut and complex semidefinite programming," Mathematical Programming, vol. 149, no. 1-2, pp. 47-81, 2015.

[10] Praneeth Netrapalli, Prateek Jain, and Sujay Sanghavi, "Phase retrieval using alternating minimization," in $A d$ vances in Neural Information Processing Systems, 2013, pp. 2796-2804.

[11] Emmanuel J Candes, Xiaodong Li, and Mahdi Soltanolkotabi, "Phase retrieval via wirtinger flow: Theory and algorithms," IEEE Transactions on Information Theory, vol. 61, no. 4, pp. 1985-2007, 2015.

[12] Yuxin Chen and Emmanuel Candes, "Solving random quadratic systems of equations is nearly as easy as solving linear systems," in Advances in Neural Information Processing Systems, 2015, pp. 739-747.
[13] Jinho Baik, Gérard Ben Arous, Sandrine Péché, et al., "Phase transition of the largest eigenvalue for nonnull complex sample covariance matrices," The Annals of Probability, vol. 33, no. 5, pp. 1643-1697, 2005.

[14] Thibault Lesieur, Florent Krzakala, and Lenka Zdeborová, "Mmse of probabilistic low-rank matrix estimation: Universality with respect to the output channel," in Communication, Control, and Computing (Allerton), 2015 53rd Annual Allerton Conference on. IEEE, 2015, pp. 680-687.

[15] Amelia Perry, Alexander S Wein, Afonso S Bandeira, and Ankur Moitra, "Optimality and sub-optimality of pca for spiked random matrices and synchronization," arXiv preprint arXiv:1609.05573, 2016.

[16] Thibault Lesieur, Florent Krzakala, and Lenka Zdeborová, "Constrained low-rank matrix estimation: Phase transitions, approximate message passing and applications," Journal of Statistical Mechanics: Theory and Experiment, vol. 2017, no. 7, pp. 073403, 2017.

[17] Yue M Lu and Gen Li, "Phase transitions of spectral initialization for high-dimensional nonconvex estimation," arXiv preprint arXiv:1702.06435, 2017.

[18] Marco Mondelli and Andrea Montanari, "Fundamental limits of weak recovery with applications to phase retrieval," arXiv preprint arXiv:1708.05932, 2017.

[19] Stefan Rotter and Sylvain Gigan, "Light fields in complex media: Mesoscopic scattering meets wave control," Reviews of Modern Physics, vol. 89, no. 1, pp. 015005, 2017.

[20] Ivo M Vellekoop and AP Mosk, "Focusing coherent light through opaque strongly scattering media," Optics letters, vol. 32, no. 16, pp. 2309-2311, 2007.

[21] Roarke Horstmeyer, Haowen Ruan, and Changhuei Yang, "Guidestar-assisted wavefront-shaping methods for focusing light into biological tissue," Nature photonics, vol. 9, no. 9, pp. 563, 2015.

[22] Elbert G van Putten, A Lagendijk, and AP Mosk, "Optimal concentration of light in turbid materials," JOSA $B$, vol. 28, no. 5, pp. 1200-1203, 2011.

[23] Ori Katz, Eran Small, Yefeng Guan, and Yaron Silberberg, "Noninvasive nonlinear focusing and imaging through strongly scattering turbid layers," Optica, vol. 1, no. 3, pp. 170-174, 2014.

[24] SM Popoff, G Lerosey, R Carminati, M Fink, AC Boccara, and S Gigan, "Measuring the transmission matrix in optics: an approach to the study and control of light propagation in disordered media," Physical review letters, vol. 104, no. 10, pp. 100601, 2010. 